\title{
Impact of frailty on surgery for glioblastoma: a critical evaluation of patient outcomes and caregivers' perceptions in a developing country
}

\author{
*Varidh Katiyar, MBBS, Ravi Sharma, MCh, Vivek Tandon, MCh, Revanth Goda, MS, \\ Akshay Ganeshkumar, MBBS, Ashish Suri, MCh, P. Sarat Chandra, MCh, \\ and Shashank S. Kale, MCh
}

Department of Neurosurgery, All India Institute of Medical Sciences, New Delhi, India

\begin{abstract}
OBJECTIVE The authors aimed to evaluate the impact of age and frailty on the surgical outcomes of patients with glioblastoma (GBM) and to assess caregivers' perceptions regarding postdischarge care and challenges faced in the developing country of India.

METHODS This was a retrospective study of patients with histopathologically proven GBM from 2009 to 2018. Data regarding the clinical and radiological characteristics as well as surgical outcomes were collected from the institute's electronic database. Taking Indian demographics into account, the authors used the cutoff age of 60 years to define patients as elderly. Frailty was estimated using the 11-point modified frailty index (mFl-11). Patients were divided into three groups: robust, with an $\mathrm{mFI}$ score of 0 ; moderately frail, with an $\mathrm{mFI}$ score of 1 or 2; and severely frail, with an $\mathrm{mFI}$ score $\geq 3$. A questionnaire-based survey was done to assess caregivers' perceptions about postdischarge care.
\end{abstract}

RESULTS Of the 276 patients, there were $93(33.7 \%)$ elderly patients and $183(66.3 \%)$ young or middle-aged patients. The proportion of severely frail patients was significantly more in the elderly group (38.7\%) than in the young or middleaged group $(28.4 \%)(p<0.001)$. The authors performed univariate and multivariate analysis of associations of different short-term outcomes with age, sex, frailty, and Charlson Comorbidity Index. On the multivariate analysis, only frailty was found to be a significant predictor for in-hospital mortality, postoperative complications, and length of hospital and ICU stay $(p<0.001)$. On Cox regression analysis, the severely frail group was found to have a significantly lower overall survival rate compared with the moderately frail $(p=0.001)$ and robust groups $(p<0.001)$. With the increase in frailty, there was a concomitant increase in the requirement for readmissions $(p=0.003)$, postdischarge specialist care $(p=0.001)$, and help from extrafamilial sources $(p<0.001)$. Greater dissatisfaction with psychosocial and financial support among the caregivers of severely frail patients was seen as they found themselves ill-equipped to provide postdischarge care at home $(p<0.001)$.

CONCLUSIONS Frailty is a better predictor of poorer surgical outcomes than chronological age in terms of duration of hospital and ICU stay, postoperative complications, and in-hospital mortality. It also adds to the psychosocial and financial burdens of the caregivers, making postdischarge care challenging.

https://thejns.org/doi/abs/10.3171/2020.7.FOCUS20482

KEYWORDS elderly; frailty; glioblastoma; surgical outcomes; caregivers' perception; end-of-life care

$\mathrm{F}$ ROM a surgeon's perspective, traditionally, the age of the patient has been an important variable in decision making as the rates of complications and adverse events following major surgeries are significantly higher in the geriatric population than in the younger population. ${ }^{1,2}$ However, there is a brewing debate regarding the roles of chronological age versus biological age in determining postoperative outcomes. ${ }^{3,4}$ Frailty has gained rec- ognition as a tool to predict the biological age, and hence, an individual's vulnerability to adverse outcomes following surgery. The use of frailty as a preoperative risk assessment model in different surgical specialties, including spine surgery, has gained popularity. However, most of the literature regarding the impact of frailty on postoperative outcomes is from the developed nations. 5,6

There is a paucity of literature on the impact of frailty

ABBREVIATIONS CCI = Charlson Comorbidity Index; DVT = deep venous thrombosis; GBM = glioblastoma; GOS-E = Glasgow Outcome Scale-Extended; KPS = Karnofsky Performance Status; $\mathrm{mFI}-11$ = 11-point modified frailty index; PE = pulmonary embolus; $\mathrm{VAP}=$ ventilator-associated pneumonia.

SUBMITTED May 31, 2020. ACCEPTED July 20, 2020.

INCLUDE WHEN CITING DOI: 10.3171/2020.7.FOCUS20482.

${ }^{*}$ V.K. and R.S. contributed equally to this work. 
in cranial neurosurgery even from the developed world. ${ }^{7}$ In developing nations, the already poor financial status of caregivers compounds the challenges of caring for patients whose condition is made more precarious by frailty. Glioblastoma (GBM), as the most common primary intracranial tumor in the geriatric population, has special relevance in this regard because treatment must be tailored for each patient. ${ }^{8}$ In view of the poor neurological status and continued need for medical attention in GBM patients, postdischarge care forms an important component in the management of this disease, ${ }^{9}$ but in many areas of developing nations there is an absence of any system similar to hospice care for the palliative treatment of terminally ill patients. In this study, which is to our knowledge the first of its kind from a developing nation, we attempted to assess the role of preoperative frailty to predict the risk of morbidity and mortality following surgery in patients with GBM. We also attempted to study the impact of preoperative frailty on postdischarge care, with special emphasis on challenges faced in the developing countries.

\section{Methods}

This was a retrospective study of patients $\geq 18$ years of age with histopathologically proven GBM who underwent biopsy or resection as the primary intervention during the period from 2009 to 2018 at the Department of Neurosurgery, All India Institute of Medical Sciences, New Delhi, India. Due clearance was provided by the Institute Ethics Committee. Patients with nonlobar GBM, multicentric GBM, or recurrent disease at presentation were excluded. Patients with incomplete clinical or radiological follow-up were also excluded. Data regarding patient characteristics, clinical features, comorbidities, radiology, postoperative course, and long-term outcomes were collected from the electronic database. All patients received postoperative adjuvant therapy depending on age, performance status, comorbidities, and extent of resection, as was determined by a multidisciplinary neuro-oncology team. Information regarding postdischarge care, psychosocial support, and financial challenges faced by the caregivers was sought by means of outpatient and/or telephone interviews using a structured questionnaire after due consent was obtained (Supplementary Material).

Usually, the age of 65 years is used as a cutoff for defining the elderly population in developed countries like the United States, where the average life expectancy is $>75$ years. However, developing countries like India have a lower life expectancy (69.73 years per 2019 statistics). ${ }^{10}$ Hence, the cutoff for the geriatric age group in India is 60 years. Considering these factors, we divided our study population into two groups (elderly and young or middle-aged patients) using the cutoff age of 60 years rather than 65 years.

Frailty was estimated using the 11-point modified frailty index (mFI-11) $)^{11,12}$ as detailed in Table 1 . The mFI-11 is a validated abridged version of the 70-point frailty index developed by Rockwood et al..$^{13}$ Patients were divided into three groups (robust, moderately frail, and severely frail) according to their preoperative mFI-11 scores as follows: 0 , robust; 1 or 2 , moderately frail; and $\geq 3$, severely frail. These cutoffs were decided based on a previous study
TABLE 1. Summary of mFI-11 components ${ }^{11,12}$

\begin{tabular}{l}
\hline \multicolumn{1}{c}{$\mathrm{mFI}-11$ Components } \\
\hline History of diabetes mellitus \\
\hline Not independent in functional status \\
\hline History of COPD or pneumonia \\
\hline History of congestive heart failure \\
\hline Myocardial infarction \\
\hline History of percutaneous coronary intervention, cardiac surgery, or \\
angina \\
\hline Hypertensive medication \\
\hline Peripheral vascular disease or rest pain \\
\hline Impaired sensorium \\
\hline Transient ischemic attack or cerebrovascular accident \\
\hline Cerebrovascular accident with deficit \\
\hline
\end{tabular}

COPD = chronic obstructive pulmonary disease .

One point is given for each of the above and a cumulative point score is calculated.

evaluating the impact of frailty on GBM patients. ${ }^{14}$ The comorbidity burden was quantified using the age-adjusted Charlson Comorbidity Index (CCI). ${ }^{15}$

The patients were assessed for short-term outcomes, including postoperative complications, in-hospital mortality, Glasgow Outcome Scale-Extended (GOS-E) score at discharge, and lengths of hospital and ICU stays. Postoperative complications were classified based on the Glioma Outcome Project System by Chang et al. ${ }^{16}$ into neurological: transient or permanent motor deficit, speech deficits; regional: wound infection, meningitis, seizures, intracerebral hemorrhage; and systemic: deep venous thrombosis (DVT) or pulmonary embolus (PE), sepsis, and ventilatorassociated pneumonia (VAP). These complications were analyzed in relation to frailty groups. The long-term outcome was assessed in terms of overall survival.

\section{Statistical Analysis}

The data were entered using Microsoft Excel 2011 and statistical analysis was done using IBM SPSS Statistics package version 20 (IBM Corp.). Kaplan-Meier plots were made using RStudio with additional packages like ggplot2, survival, survminer, dplyr, and tidyr. Chi-square test, Fischer exact test, independent-samples KruskalWallis test, Student t-test, and simple and multiple logistic regression were used as appropriate. For survival data, a multivariate-adjusted Cox proportional hazards model was constructed. For all statistical tests, a p value $<0.05$ was considered to be statistically significant.

\section{Results}

A total of 276 patients met the eligibility criteria and were included in the study. The study population was divided according to chronological age into two groups, elderly and young or middle-aged, with a cutoff age of 60 years. Of the 276 patients, there were 93 (33.7\%) elderly and $183(66.3 \%)$ young or middle-aged patients. Comparisons of various clinical characteristics between elderly 
TABLE 2. Comparison of clinical characteristics of elderly and young or middle-aged GBM patients

\begin{tabular}{|c|c|c|c|c|}
\hline Characteristic & Total & $\begin{array}{l}\text { Elderly } \\
(n=93)\end{array}$ & $\begin{array}{l}\text { Young or Middle-Aged } \\
\qquad(n=183)\end{array}$ & $\mathrm{p}$ Value \\
\hline Sex & & & & 0.039 \\
\hline Male & $191(69.2 \%)$ & $72(77.4 \%)$ & $119(65 \%)$ & \\
\hline Female & $85(30.8 \%)$ & $21(22.6 \%)$ & $64(35 \%)$ & \\
\hline Mean $\mathrm{BMI} \pm \mathrm{SD}, \mathrm{kg} / \mathrm{m}^{2}$ & $23.69 \pm 3.58$ & $23.62 \pm 3.90$ & $23.73 \pm 3.41$ & 0.806 \\
\hline Admission GCS score & 15 [12-15] & 15 [13-15] & 15 [12-15] & 0.546 \\
\hline $\mathrm{mFI}-11$ score & & & & 0.008 \\
\hline Overall & $2[0-3]$ & $2[1-4]$ & $1[0-3]$ & \\
\hline Robust (score 0) & $80(29 \%)$ & $16(17.2 \%)$ & $64(35 \%)$ & \\
\hline Moderately frail (score 1-2) & $108(39.1 \%)$ & $41(44.1 \%)$ & $67(36.6 \%)$ & \\
\hline Severely frail (score >2) & $88(31.9 \%)$ & $36(38.7 \%)$ & $52(28.4 \%)$ & \\
\hline Diabetes mellitus & $51(18.5 \%)$ & $24(25.8 \%)$ & $27(14.8 \%)$ & 0.033 \\
\hline Hypertension & $88(31.9 \%)$ & $43(46.2 \%)$ & $45(24.6 \%)$ & $<0.001$ \\
\hline Preop KPS score & $50[30-80]$ & $50[30-65]$ & $50[30-80]$ & 0.102 \\
\hline $\mathrm{CCl}$ score & $2[1-4]$ & $4[3-5]$ & $2[0-2]$ & $<0.001$ \\
\hline Postop GCS score & $15[11-15]$ & $15[13-15]$ & $15[11-15]$ & 0.434 \\
\hline Tracheostomy due to prolonged ventilation & $50(18.1 \%)$ & $16(17.2 \%)$ & $34(18.6 \%)$ & 0.779 \\
\hline \multicolumn{5}{|l|}{ Complications } \\
\hline Overall & $142(51.4 \%)$ & $53(57 \%)$ & $89(48.6 \%)$ & 0.204 \\
\hline Neurological & $119(43.1 \%)$ & $44(47.3 \%)$ & $75(41 \%)$ & 0.368 \\
\hline \multicolumn{5}{|l|}{ Regional } \\
\hline Seizures & $36(13.1 \%)$ & $13(14.1 \%)$ & $23(12.6 \%)$ & 0.709 \\
\hline Wound infection & $31(11.3 \%)$ & $12(13 \%)$ & $19(10.4 \%)$ & 0.547 \\
\hline Meningitis & $38(13.8 \%)$ & $12(13 \%)$ & $26(14.2 \%)$ & 0.855 \\
\hline Intracranial bleed & $68(24.7 \%)$ & $22(23.9 \%)$ & $46(25.1 \%)$ & 0.883 \\
\hline \multicolumn{5}{|l|}{ Systemic } \\
\hline DVT/PE & $5(1.8 \%)$ & $4(4.3 \%)$ & $1(0.5 \%)$ & 0.04 \\
\hline VAP & $54(19.6 \%)$ & $22(23.9 \%)$ & $32(17.5 \%)$ & 0.26 \\
\hline Sepsis & $89(32.4 \%)$ & $33(35.9 \%)$ & $56(30.6 \%)$ & 0.413 \\
\hline Duration of ICU stay, days & $2[1-7]$ & $3[1-7]$ & $2[1-7]$ & 0.74 \\
\hline Duration of hospital stay, days & $5[4-11]$ & $6[3-11]$ & $5[4-11]$ & 0.94 \\
\hline In-hospital mortality & $21(7.6 \%)$ & $7(7.5 \%)$ & $14(7.7 \%)$ & 1.00 \\
\hline GCS score at discharge & $15[13-15]$ & $15[14-15]$ & $15[12-15]$ & 0.712 \\
\hline GOS-E score at discharge & $5[4-7]$ & $4[4-6]$ & $5[4-7]$ & $<0.001$ \\
\hline Surgery for recurrent disease & $49(17.8 \%)$ & $9(9.7 \%)$ & $40(21.9 \%)$ & $<0.001$ \\
\hline Overall survival & $11[5-17]$ & $10[4-14]$ & $13[6-18]$ & 0.035 \\
\hline
\end{tabular}

Elderly = age $\geq 60$ years; $G C S=$ Glasgow Coma Scale; young or middle-aged $=$ age $<60$ years .

Values are presented as the number (\%) of patients or median [IQR] unless otherwise indicated.

and young or middle-aged GBM patients are summarized in Table 2.

Using the mFI-11 score, we also divided the patients based on their frailty into robust $(\mathrm{mFI}=0)$, moderately frail $(0<\mathrm{mFI}<3)$, and severely frail $(\mathrm{mFI} \geq 3)$ groups. There were 80 robust (29.0\%), 108 moderately frail (39.1\%), and 88 severely frail (31.9\%) patients in our cohort.

\section{Association of Biological Age (Frailty) With Chronological Age}

The frailty scores were found to be positively corre- lated with increasing age $(\mathrm{p}=0.008)$ (Fig. 1A). Similarly, the proportion of severely frail patients was significantly greater in the elderly group $(38.7 \%)$ than in the young or middle-aged group $(28.4 \%, \mathrm{p}<0.001)$ (Fig. 1B).

\section{Surgical Decision Making}

\section{Association of Frailty With Decision to Perform Biopsy}

The total number of patients who underwent biopsy was 7 , all of whom were severely frail ( $p<0.001)$, while all of the patients in the moderately frail and robust groups 

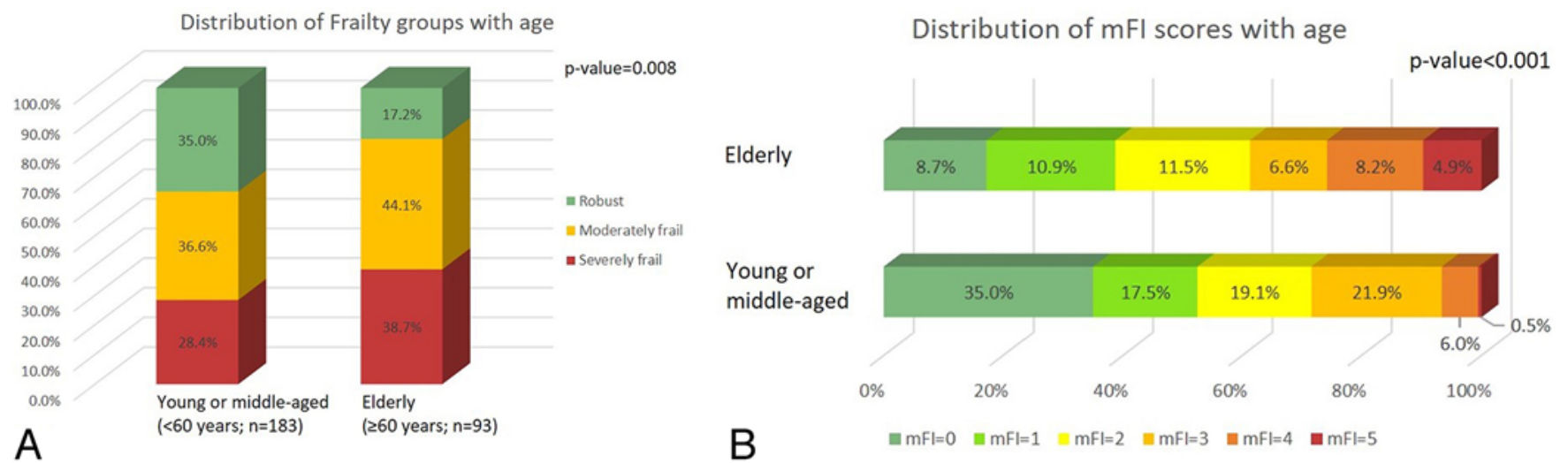

FIG. 1. Distribution of $\mathrm{mFI}-11$ scores (A) and frailty categories (B) according to chronological age.

were planned for resection. However, even among the severely frail patients an attempt at resection (81 patients, $92.1 \%$ ) was preferred over biopsy (7 patients, $7.9 \%$ ).

\section{Association of Frailty With Decision to Perform Resection for Recurrent/Residual Disease}

Of the 108 patients who were diagnosed with recurrent/ residual disease, the severely frail patients were less likely to undergo resection (8 of 23 patients, $34.7 \%$ ) than the patients in the moderately frail (21 of 47 patients, $44.6 \%$ ) and robust (20 of 38 patients, $52.6 \%$ ) groups; however, the difference failed to reach statistical significance $(p=0.39)$.

\section{Postoperative Adjuvant Therapy}

Of the 276 patients who underwent surgery, 21 died during the hospital stay. The postoperative adjuvant therapy was individualized for each of the surviving 255 patients by a combined neuro-oncology team taking into consideration the age, postoperative Karnofsky Performance Status (KPS) score, MGMT methylation status, comorbidities, and willingness of the patient or their caregivers. Twenty-six patients $(10.2 \%)$ received only best supportive care. Of the remaining 229 patients, 47 patients received adjuvant therapy at peripheral medical centers due to the long waiting list at our center. Details regarding the nature of adjuvant therapy of these patients was not available owing to the retrospective nature of study. In total, 182 patients received adjuvant therapy at our center. Of these, 141 patients $(77.5 \%)$ received concurrent chemoradiation (temozolomide [TMZ] + 60 Gy radiation therapy [RT] in 30 fractions) followed by TMZ. Fifteen patients (8.2\%) received TMZ alone while 22 patients $(12.1 \%)$ received hypofractionated RT alone (30-45 Gy in 10-15 fractions). Four patients (2.2\%) received both TMZ and hypofractionated RT. The type of adjuvant therapy administered showed a trend toward greater likelihood of concurrent chemoradiotherapy among the robust and moderately frail compared to the severely frail group; however, the difference failed to reach statistical significance $(p=0.06)$.

\section{Predictors of Short-Term Outcomes}

We performed univariate analysis of possible associations of different surgical outcomes with age, sex, frailty, and CCI score. On univariate analysis, frailty was found to be a significant predictor for in-hospital mortality, postoperative complications, and length of hospital and ICU stay $(\mathrm{p}<0.001)$. CCI score correlated significantly only with the rate of postoperative complications $(\mathrm{p}<0.001)$. On multivariate analysis, only frailty was found to be a significant predictor across all of the above outcomes, while CCI score lost its significance for predicting postoperative complications $(\mathrm{p}=0.758)$.

\section{Correlation of Frailty With Postoperative Complications}

Patients in the severely frail group were found to be more predisposed to postoperative regional and systemic complications $(\mathrm{p}<0.001)$. In this group, approximately 80 of 88 patients $(90.9 \%)$ suffered from some type of postoperative complications, 64 patients (72.7\%) suffered regional complications, and 76 patients (86.4\%) suffered systemic complications (Fig. 2A). On evaluating the in-hospital mortality associated with the primary admission among the patients with different levels of frailty, we found similar results. Of 88 severely frail patients, 16 patients (18.2\%) succumbed in the postoperative period compared to $3.7 \%$ of the moderately frail and $1.3 \%$ of the robust patients, as shown in Fig. 2B. Of the 255 surviving patients at discharge, the median GOS-E score was significantly lower in the severely frail patients (4, IQR 4-4.5) compared to the moderately frail (5, IQR 4-6) and robust (7, IQR 7-7) patients $(\mathrm{p}<0.001)$.

The proportion of patients who underwent tracheostomy was also significantly higher in the frailer groups. It was observed that 37 of 88 severely frail patients $(42.1 \%)$ underwent tracheostomy, while the proportion of patients who needed tracheostomy was $13.9 \%$ in the moderately frail and just $1.25 \%$ in the robust patients $(\mathrm{p}<0.001)$.

\section{Association of Frailty With Lengths of Hospital and ICU Stays}

The lengths of hospital and ICU stays were found to be significantly correlated with the level of frailty. The median duration of hospital stay was significantly longer among the severely frail patients (12 days; IQR 7-16 days) than the moderately frail (5 days; IQR 3-7 days) and robust (4 days; IQR 3-5.5 days) patients ( $\mathrm{p}<0.001)$. Similarly, the median duration of ICU stay was also found to be significantly lon- 
Complication rates with respect to frailty groups

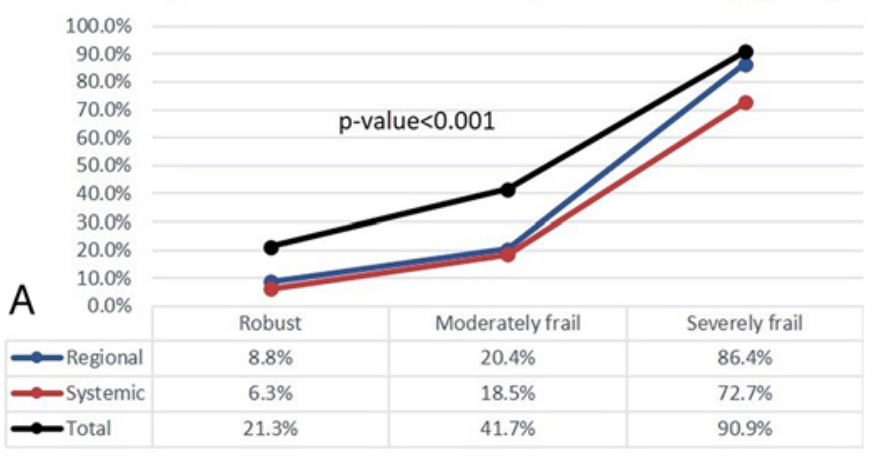

In-hospital mortality with respect to frailty groups

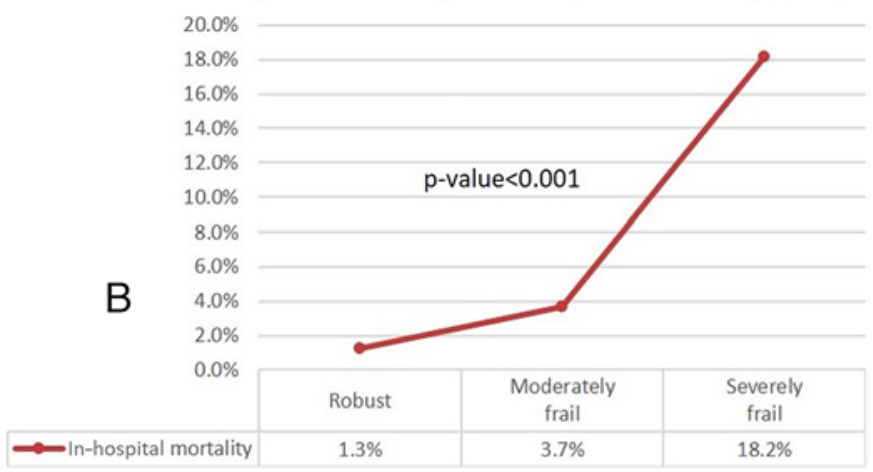

FIG. 2. Comparison of postoperative complications (A) and in-hospital mortality (B) among frailty categories.

ger in the severely frail (8.5 days; IQR 4-13 days) than in the moderately frail (2 days; IQR 1-2 days) and robust (2 days; IQR $1-2$ days) patients ( $p<0.001)$. The time trends of discharge from ICU to ward and further discharge to home have been summarized in Fig. 3A and B.

\section{Predictors of Long-Term Outcome \\ Overall Survival}

The overall survival was found to be independently associated with the level of frailty as well as chronological age. On multivariate Cox regression analysis, the severely frail group was found to have a significantly lower overall survival than the moderately frail (HR 1.75; 95\% CI 1.232.5 ) and robust (HR 1.83; 95\% CI 1.26-2.68) groups. Similarly, the elderly group had lower overall survival than the young or middle-aged group (HR 1.43; 95\% CI 1.06-1.95). The Kaplan-Meier survival curves for the different levels of frailty are shown in Fig. 3C (log-rank test, $p=0.037$ ).

\section{Impact of Frailty on Caregiver's Perceptions of End-of-Life Care in GBM}

Of the total study population, caregivers of 181 patients gave consent for participation in an end-of-life care survey. The frailty of the patients was also found to have an impact on the postdischarge care of the patients and the consequent impact on the caregivers. The moderately and severely frail patients were found to require care in local hospitals and sometimes the primary hospital during the last month of life more frequently than their robust counterparts $(\mathrm{p}<0.001)$. The requirement for readmissions to the primary hospital after discharge showed a similar trend. Similarly, the need for postdischarge specialist care from a neurosurgeon or an oncologist was encountered more among the severely frail $(63.6 \%)$ patients than the moderately frail $(46.2 \%)$ and robust $(30 \%)$ patients. The caregiver's need to seek help from extrafamilial sources like social acquaintances, maids, or medically accredited nurses was also found to be significantly greater with increasing level of patient frailty (Table 3).

The caregiver's satisfaction with the surgical treatment received by the patient during the primary admission remained high across all the groups of frailty (Fig. 4A). There was a trend toward greater dissatisfaction with psy- chosocial support among the caregivers of severely frail patients; however, it failed to reach statistical significance ( $p=0.20)$ (Fig. 4B). As the frailty increased, the caregivers found themselves unequipped to provide postdischarge care at home $(\mathrm{p}<0.001)$ (Fig. 4C). Caregivers of severely frail and moderately frail patients also reported facing a greater financial burden imposed by the postdischarge care of the patient $(\mathrm{p}<0.001)$ (Fig. 4D).

\section{Discussion}

GBM carries a poor prognosis, which can be attributed to the malignant nature of the disease, the frail nature of the predominantly elderly patient population, and the molecular patterns of the disease. ${ }^{17,18}$ Most of the studies in the literature have focused primarily on surgical factors like the extent of resection for understanding the prognosis and survival of patients with GBM. However, considering the advanced age and comorbidities of the patients, surgery as well as the postoperative course can be challenging. To evaluate the impact of these premorbid patient characteristics, the majority of the researchers have used variables such as age, functional status, presence of neurological deficits, etc., with mixed results. ${ }^{7,19,20}$ However, the concept of biological age, i.e., frailty, has gained popularity among different medical and surgical specialties as well as in spine surgery. Assessment of patient frailty has emerged as an important tool in predicting the mortality, morbidity, and length of the hospital stay in the elderly population. ${ }^{14,21-23}$

Different studies have used different age cutoffs to define the elderly population and assess the impact of frailty on surgical outcomes..$^{24,25}$ The rationale to use a cutoff age of 60 years to define the geriatric population in our study is based on a lower life expectancy in our country compared to developed nations.

In our study, the incidence of severe frailty was $38.7 \%$ of the elderly population, whereas in the studies by Cloney et al. ${ }^{14}$ and Giaccherini et al. ${ }^{26}$ the incidence rates were $19.3 \%$ and $44.1 \%$, respectively. The higher proportion of frailty in our study, despite the lower cutoff age used to define "elderly," can be explained by considering the difference in the race and socioeconomic status of our population. In a study conducted by Asemota and Gallia, ${ }^{21}$ the authors' finding that frailty rates were higher in patients 

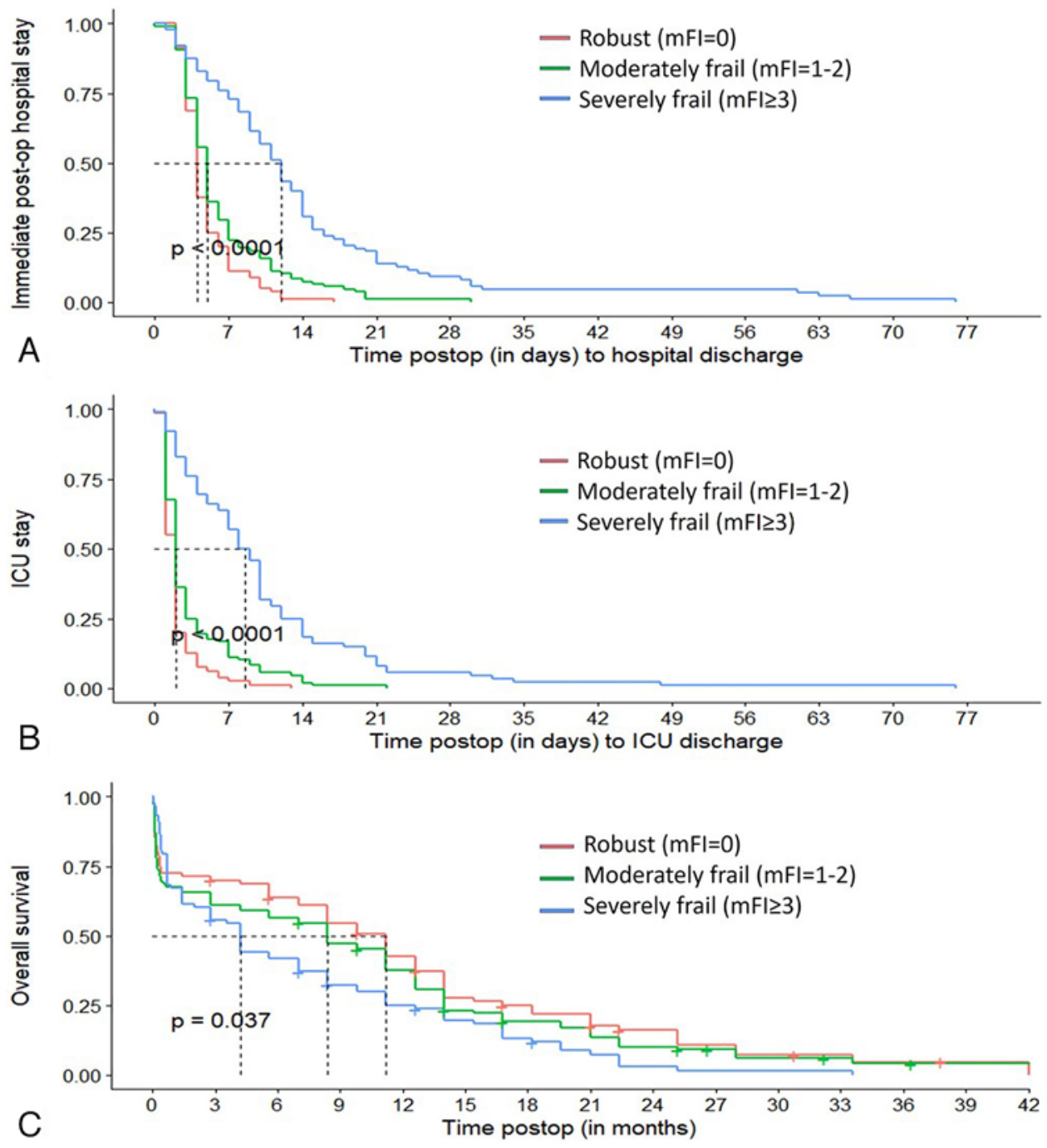

FIG. 3. Kaplan-Meier survival curves comparing postoperative hospital stay (A), ICU stay (B), and overall survival (C) among the different categories of frailty (log-rank test).

of low- to medium-income groups lends support to this hypothesis. On the other hand, the higher rate of frailty reported by Giaccherini et al. ${ }^{26}$ may be attributed to the different definitions of frailty used.

In the geriatric population, surgical decision making in cranial surgeries is complex and is influenced by the chronological age and comorbidities of the patient and often comes down to the surgeon's decision and the willingness of the patient, which are arrived at subjectively. ${ }^{1,2,27} \mathrm{~A}$ simple yet accurate objective tool is imperative to guide the treatment in such patients, because the use of only chronological age to determine frailty is fraught with error. In oncological neurosurgery, the most commonly used tools are standardized assessments such as the American Society of Anesthesiologists (ASA) score, Acute Physiology and Chronic Health Evaluation (APACHE-II), Physiological and Severity Score for the Enumeration of Mortality and Morbidity (POSSUM), and the prognostic nutritional index (PNI). While the KPS assessment does not consider any comorbidities, the ASA score is subjective, and
APACHE-II and POSSUM are cumbersome to calculate at the bedside and thus are not useful to guide treatment in the geriatric population. ${ }^{7,28}$ Thus, there has been a vacuum in terms of a simple yet effective tool for decision making. Frailty, widely considered to be an indicator of biological age, has been reported in the literature to perform well in this regard not only in other surgical specialties but in spine surgery as well. ${ }^{29}$ One of the most commonly used frailty indices, the mFI-11, may prove to be an important bedside tool for this purpose by providing an objective alternative to the more subjective decision-making process being utilized currently in clinical practice.

We found that frailty is associated with increased hospital and ICU stay durations as well as with lower overall survival in GBM patients. Dasgupta et al. ${ }^{30}$ and Makary et al. ${ }^{31}$ also concluded that frailty is an independent predictor of length of hospital stay following surgery, irrespective of chronological age.

In our study, we used the Glioma Outcome Project system proposed by Chang et al. ${ }^{16}$ to classify the postopera- 
TABLE 3. Comparison of end-of-life care characteristics between robust, moderately frail, and severely frail GBM patients

\begin{tabular}{llcccc}
\hline End-of-Life Care Characteristics & Total $(n=181)$ & Robust $(n=50)$ & Moderately Frail $(n=65)$ & Severely Frail $(n=66)$ & $p$ Value \\
\hline Place & & & & & $<0.001$ \\
\hline Primary hospital & $28(15.4 \%)$ & $3(6 \%)$ & $10(15.38 \%)$ & $15(22.72 \%)$ & \\
\hline Local hospital & $83(45.8 \%)$ & $9(18 \%)$ & $33(50.76 \%)$ & $41(62.12 \%)$ & \\
\hline Home & $70(38.6 \%)$ & $38(76 \%)$ & $22(33.84 \%)$ & $10(15.15 \%)$ & \\
\hline Need for specialist care & $87(48.06 \%)$ & $15(30 \%)$ & $30(46.15 \%)$ & $42(63.64 \%)$ & 0.001 \\
\hline Rehospitalization & $38(21.0 \%)$ & $5(10 \%)$ & $13(20 \%)$ & $20(30.3 \%)$ & 0.03 \\
\hline Primary extrafamilial help* & $93(51.38 \%)$ & $18(36 \%)$ & $27(41.54 \%)$ & $48(72.73 \%)$ & $<0.001$ \\
\hline
\end{tabular}

Values are presented as the number (\%) of patients unless otherwise indicated.

* Social acquaintance, maid, and medically accredited nurses.

tive complications into systemic, local, and neurological complication categories. The rates of complications were observed to be higher in the severely frail group than the robust population, a finding which is in consensus with the existing literature. ${ }^{14}$

\section{Caregiver's satisfaction with surgical treatment}

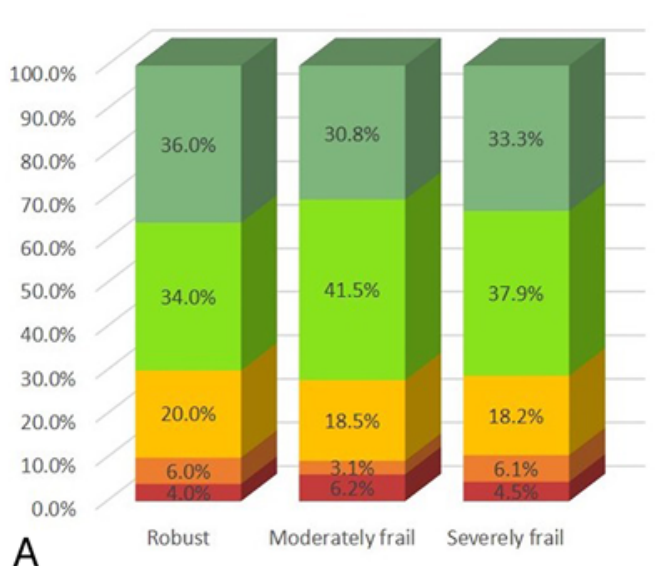

p-value $=0.98$

Caregiver's perception about their ability to provide postdischarge care
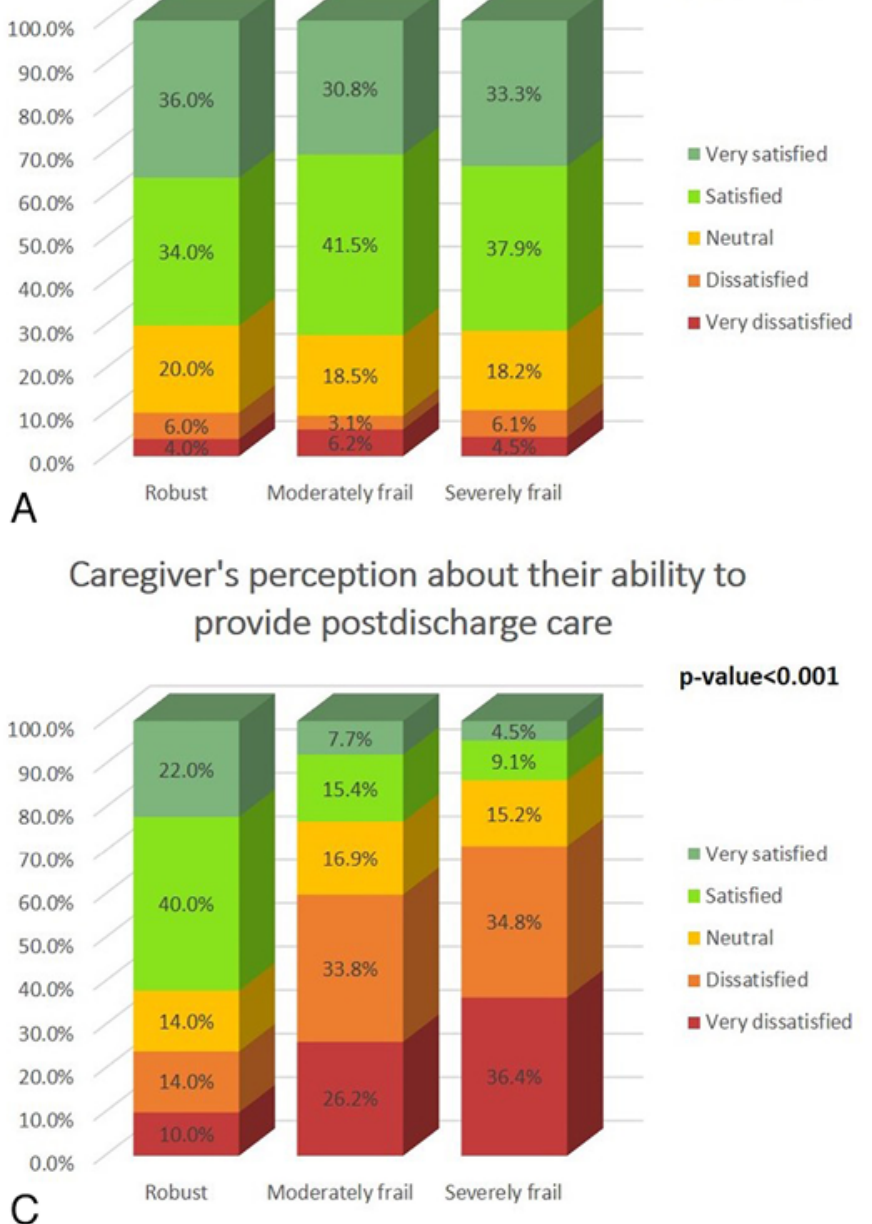

value<0.001
Our study showed an overall median survival period following surgery of 11 months, which is slightly less than that reported elsewhere in the literature ${ }^{32-35}$ Keeping the predominant socioeconomic strata of patients in mind, the lower overall survival may be attributable to the lack

\section{Caregiver's satisfaction with psychosocial support}

$\begin{array}{r}100.0 \% \\ 90.0 \% \\ 80.0 \% \\ 70.0 \% \\ 60.0 \% \\ 50.0 \% \\ 40.0 \% \\ \hline 30.0 \% \\ \hline 20.0 \% \\ 10.0 \% \\ \hline 0.0 \% \\ \hline \text { B }\end{array}$
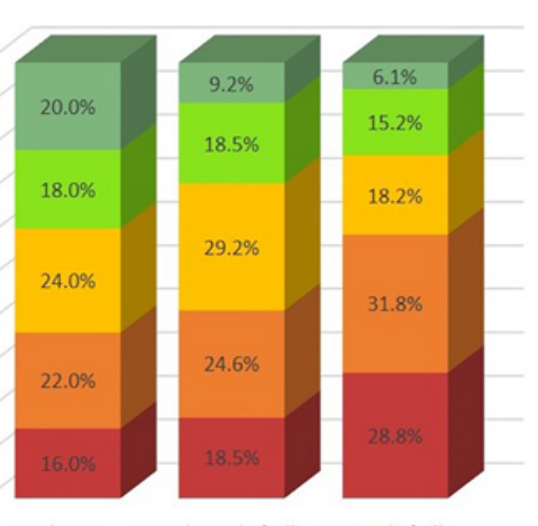

p-value $=0.20$

Robust Moderately frail Severely frail

Financial burden due to postdischarge care

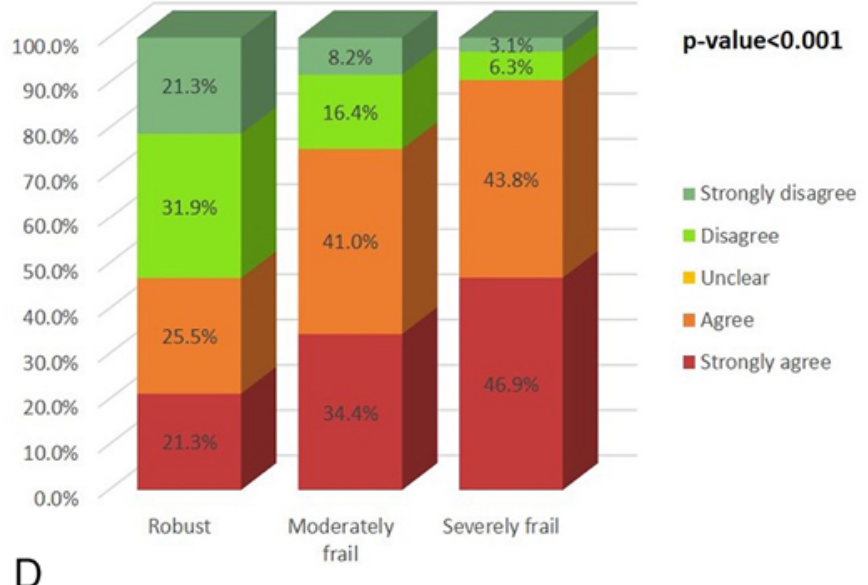

FIG. 4. Caregiver satisfaction with surgical treatment (A) and psychosocial support (B); and caregiver perception regarding ability to provide postdischarge care at home (C) and financial burden imposed by postdischarge care (D). 
of proper care of patients following discharge from the hospital, combined with the lack of any hospice system in developing countries compared with the availability of such a system in Western countries. In the absence of any established system, social acquaintances and maids form the support system for the caregivers in lower- to middleincome countries. The greater need for postdischarge care for GBM patients, compounded further by frailty, makes the dependence on such extrafamilial sources imperative.

According to our survey on caregivers' perceptions, the increasing level of frailty significantly added to the already taxed financial condition of the patients' families. Poor socioeconomic status often prevents the caregivers from providing adequate end-of-life care. Caregivers had also reported that patients require more rehospitalizations with increasing levels of frailty. These findings warrant the need for establishment of local nursing facilities or hospice centers for better care, providing financial assistance for caregivers, and developing appropriate infrastructure at the rural level for better end-of-life care of terminally ill patients.

The previously existing literature assessing the predictors of neurosurgical outcomes has primarily focused on patient chronological age. Frailty, which is widely accepted as a marker for biological age, has much more clinical significance in determining surgical outcomes than chronological age..$^{36}$ Our study adds to the growing body of evidence that chronological age may just be a number and frailty is a better predictor of surgical outcomes. Hence, robust patients should not be deprived of the benefit of aggressive surgical management, irrespective of their age.

Our study is unique as it is to our knowledge the first study conducted in the developing world to assess the impact of frailty on surgical outcomes of GBM patients. This report adds to the existing scarce literature dealing with the role of frailty in cranial neurosurgery. We have also described the impact of frailty on the caregiver's perceptions regarding end-of-life care. The special challenges faced by the patients and their caregivers in resource-constrained settings have been highlighted.

This study has some limitations owing to its retrospective design. As the apex tertiary care center of our country, our facility receives more patients than can be accommodated in existing healthcare facilities. Due to long waiting lists at our center, some of our patients underwent adjuvant therapy at peripheral medical centers. The data regarding the type of adjuvant therapy for these patients were thus incomplete. Although we found a trend in favor of less frail patients receiving concurrent chemoradiotherapy, currently the decision regarding the type of adjuvant therapy is usually based on chronological age and postoperative KPS score. We feel that postoperative frailty needs to be evaluated as a criterion to decide the form of adjuvant therapy in future studies.

In different studies of this topic, there is significant heterogeneity not only in the various scores assessing frailty but also in the categorization cutoffs used. ${ }^{37}$ Also, all such scores have been validated in the developed world. To address these concerns, specifically with regard to developing countries, we are doing a prospective study to validate the $\mathrm{mFI}-11$ scale for assessing frailty in the Indian popula- tion. Since the current study is a single-center study conducted at a tertiary care center, it may not be representative of frailty trends and their impact across the entire country, a concern that highlights the need for a national database of preoperative risk assessment variables in our country. Thus, a large prospective multicenter study is warranted to evaluate the effects of preoperative frailty on surgical outcomes and postdischarge care, particularly in developing countries.

\section{Conclusions}

The biological age of the patient as manifested by frailty is a better predictor than chronological age of poorer surgical outcomes in terms of durations of hospital and ICU stays, postoperative complications, and in-hospital mortality in patients undergoing surgery for GBM. Frailty also adds to the psychosocial and financial burden of the caregivers. An attempt should be made to evaluate the frailty of GBM patients before surgical intervention for better surgical decision making and counseling regarding postdischarge care.

\section{Acknowledgments}

We would like to thank Dr. Simranjeet Singh for his valuable contribution in data collection.

\section{References}

1. Sullivan LM, Massaro JM, D’Agostino RB Sr. Presentation of multivariate data for clinical use: The Framingham Study risk score functions. Stat Med. 2004;23(10):1631-1660.

2. Revenig LM, Canter DJ, Taylor MD, et al. Too frail for surgery? Initial results of a large multidisciplinary prospective study examining preoperative variables predictive of poor surgical outcomes. J Am Coll Surg. 2013;217(4):665-670.e1.

3. Rockwood K, Mitnitski A. Frailty in relation to the accumulation of deficits. J Gerontol A Biol Sci Med Sci. 2007;62(7): $722-727$.

4. De Lepeleire J, Iliffe S, Mann E, Degryse JM. Frailty: an emerging concept for general practice. Br J Gen Pract. 2009; 59(562):e177-e182.

5. Bergman H, Ferrucci L, Guralnik J, et al. Frailty: an emerging research and clinical paradigm-issues and controversies. J Gerontol A Biol Sci Med Sci. 2007;62(7):731-737.

6. Fulop T, Larbi A, Witkowski JM, et al. Aging, frailty and age-related diseases. Biogerontology. 2010;11(5):547-563.

7. Reponen E, Tuominen H, Korja M. Evidence for the use of preoperative risk assessment scores in elective cranial neurosurgery: a systematic review of the literature. Anesth Analg. 2014;119(2):420-432.

8. Lorimer CF, Saran F, Chalmers AJ, Brock J. Glioblastoma in the elderly-How do we choose who to treat? J Geriatr Oncol. 2016;7(6):453-456.

9. Hertler C, Eisele G, Gramatzki D, et al. End-of-life care for glioma patients; the caregivers' perspective. J Neurooncol. 2020;147(3):663-669.

10. Verma R, Khanna P. National program of health-care for the elderly in India: a hope for healthy ageing. Int J Prev Med. 2013;4(10):1103-1107.

11. Farhat JS, Velanovich V, Falvo AJ, et al. Are the frail destined to fail? Frailty index as predictor of surgical morbidity and mortality in the elderly. J Trauma Acute Care Surg. 2012; 72(6):1526-1531.

12. Velanovich V, Antoine H, Swartz A, et al. Accumulating deficits model of frailty and postoperative mortality and mor- 
bidity: its application to a national database. J Surg Res. 2013; 183(1):104-110.

13. Rockwood K, Song X, MacKnight C, et al. A global clinical measure of fitness and frailty in elderly people. CMAJ. 2005; 173(5):489-495.

14. Cloney M, D'Amico R, Lebovic J, et al. Frailty in geriatric glioblastoma patients: a predictor of operative morbidity and outcome. World Neurosurg. 2016;89:362-367.

15. Charlson ME, Pompei P, Ales KL, MacKenzie CR. A new method of classifying prognostic comorbidity in longitudinal studies: development and validation. J Chronic Dis. 1987; 40(5):373-383.

16. Chang SM, Parney IF, McDermott M, et al. Perioperative complications and neurological outcomes of first and second craniotomies among patients enrolled in the Glioma Outcome Project. J Neurosurg. 2003;98(6):1175-1181.

17. Barnholtz-Sloan JS, Williams VL, Maldonado JL, et al. Patterns of care and outcomes among elderly individuals with primary malignant astrocytoma. J Neurosurg. 2008;108(4): 642-648.

18. Paszat L, Laperriere N, Groome P, et al. A population-based study of glioblastoma multiforme. Int J Radiat Oncol Biol Phys. 2001;51(1):100-107.

19. Schag CC, Heinrich RL, Ganz PA. Karnofsky performance status revisited: reliability, validity, and guidelines. J Clin Oncol. 1984;2(3):187-193.

20. Crooks V, Waller S, Smith T, Hahn TJ. The use of the Karnofsky Performance Scale in determining outcomes and risk in geriatric outpatients. J Gerontol. 1991;46(4):M139-M144.

21. Asemota AO, Gallia GL. Impact of frailty on short-term outcomes in patients undergoing transsphenoidal pituitary surgery. J Neurosurg. 2019;132(2):360-370.

22. Rahmani R, Tomlinson SB, Santangelo G, et al. Risk factors associated with early adverse outcomes following craniotomy for malignant glioma in older adults. J Geriatr Oncol. 2020; 11(4):694-700.

23. Tomlinson SB, Piper K, Kimmell KT, Vates GE. Preoperative frailty score for 30-day morbidity and mortality after cranial neurosurgery. World Neurosurg. 2017;107:959-965.

24. Conroy S, Dowsing T. The ability of frailty to predict outcomes in older people attending an acute medical unit. Acute Med. 2013;12(2):74-76.

25. Evans SJ, Sayers M, Mitnitski A, Rockwood K. The risk of adverse outcomes in hospitalized older patients in relation to a frailty index based on a comprehensive geriatric assessment. Age Ageing. 2014;43(1):127-132.

26. Giaccherini L, Galaverni M, Renna I, et al. Role of multidimensional assessment of frailty in predicting outcomes in older patients with glioblastoma treated with adjuvant concurrent chemo-radiation. J Geriatr Oncol. 2019;10(5): 770-778.

27. Wilson JR, Clarke MG, Ewings P, et al. The assessment of patient life-expectancy: how accurate are urologists and oncologists? BJU Int. 2005;95(6):794-798.

28. Chand M, Armstrong T, Britton G, Nash GF. How and why do we measure surgical risk? J R Soc Med. 2007;100(11): 508-512.

29. Simcox T, Antoku D, Jain N, et al. Frailty syndrome and the use of frailty indices as a preoperative risk stratification tool in spine surgery: a review. Asian Spine J. 2019;13(5):861-873.

30. Dasgupta M, Rolfson DB, Stolee P, et al. Frailty is associated with postoperative complications in older adults with medical problems. Arch Gerontol Geriatr. 2009;48(1):78-83.
31. Makary MA, Segev DL, Pronovost PJ, et al. Frailty as a predictor of surgical outcomes in older patients. J Am Coll Surg. 2010;210(6):901-908.

32. Chaichana KL, Chaichana KK, Olivi A, et al. Surgical outcomes for older patients with glioblastoma multiforme: preoperative factors associated with decreased survival. Clinical article. J Neurosurg. 2011;114(3):587-594.

33. Delgado-López PD, Corrales-García EM. Survival in glioblastoma: a review on the impact of treatment modalities. Clin Transl Oncol. 2016;18(11):1062-1071.

34. Simpson JR, Horton J, Scott C, et al. Influence of location and extent of surgical resection on survival of patients with glioblastoma multiforme: results of three consecutive Radiation Therapy Oncology Group (RTOG) clinical trials. Int $J$ Radiat Oncol Biol Phys. 1993;26(2):239-244.

35. Keles GE, Anderson B, Berger MS. The effect of extent of resection on time to tumor progression and survival in patients with glioblastoma multiforme of the cerebral hemisphere. Surg Neurol. 1999;52(4):371-379.

36. Goggins WB, Woo J, Sham A, Ho SC. Frailty index as a measure of biological age in a Chinese population. J Gerontol A Biol Sci Med Sci. 2005;60(8):1046-1051.

37. Pazniokas J, Gandhi C, Theriault B, et al. The immense heterogeneity of frailty in neurosurgery: a systematic literature review. Neurosurg Rev. Published online January 17, 2020. doi:10.1007/s10143-020-01241-2

\section{Disclosures}

The authors report no conflict of interest concerning the materials or methods used in this study or the findings specified in this paper.

\section{Author Contributions}

Conception and design: Tandon, Katiyar, Sharma. Acquisition of data: Katiyar, Goda. Analysis and interpretation of data: Katiyar, Sharma. Drafting the article: Katiyar, Sharma, Goda, Ganeshkumar. Critically revising the article: Tandon, Katiyar, Sharma, Goda, Ganeshkumar. Reviewed submitted version of manuscript: Katiyar, Sharma. Statistical analysis: Katiyar. Administrative/technical/material support: Tandon, Sharma, Suri, Chandra, Kale. Study supervision: Sharma, Suri, Chandra, Kale.

\section{Supplemental Information \\ Online-Only Content}

Supplemental material is available online.

Supplementary Material. https://thejns.org/doi/suppl/10.3171/ 2020.7.FOCUS20482.

\section{Correspondence}

Vivek Tandon: All India Institute of Medical Sciences, New Delhi, India.drtandonvivek@gmail.com. 